\title{
Interactive comment on "Seawater pH reconstruction using boron isotopes in multiple planktonic foraminifera species with different depth habitats and their potential to constrain $\mathrm{pH}$ and $\mathrm{pCO}_{2}$ gradients" by Maxence Guillermic et al.
}

Maxence Guillermic et al.

maxence.guillermic@gmail.com

Received and published: 23 October 2019

We wish to thank Dr. Jesse Farmer for his thorough review of our manuscript and his helpful comments. We believe that we addressed all of the major comments indicated by Dr. Farmer as indicated in the discussion below and the updated manuscript (see supplement). 
regions simply a function of variations in thermocline depth? This point could be clarified throughout the manuscript. If true, it seems a particularly important outcome of this study is a need to combine planktonic foraminifera _ $11 \mathrm{~B}$ with thermocline depth reconstructions.

Response 1: We have added a statement to line 460 acknowledging that "Factors including variations in thermocline depth can impact depth habitats for some taxa." Variations in thermocline depth are certainly one of several factors that impact the differences in foraminiferal depth habitats, but not the only factor, both in the present and in the past. Only some species follow the thermocline depth (like G. tumida), while others (such as N. dutertrei) are not and are usually found around the same water depth. So it will be dependent on the studied species.

Comment 2: In section 5.2 (L482-514), the authors posit a primary control of light availability on foraminiferal _11B deviations from _11Bborate. While an interesting idea, unfortunately I think the weight of this section is not supported by the authors' data. This whole discussion is essentially predicated on a single T. sacculifer _ 11B measurement from the western Equatorial Pacific, which has anomalously low _11Bforam value (relative to _11Bborate) compared to previous studies. The authors use this single observation to make a complex argument about how foraminifera calcification depth impacts light availability, which impacts symbiont photosynthesis, which affects microenvironment $\mathrm{pH}$ and hence foram _11B. Not only does this strike me as insufficient evidence to justify a discussion of this length, there are numerous assumptions and issues within the discussion that require referencing and clarification (see detailed comments below).

Response 2: We understand this point. Further support for this comes from data for ODP Sites $806 \mathrm{~A}$ and $807 \mathrm{~A}$ which we have added to this study. The Holocene data for Sites 806A and record similar low values to what we reported for site WPO7. This strengthens and justifies the length of the discussion.

Printer-friendly version

Discussion paper 
Comment 3: Section 5.4 gives a rather hasty overview of how measuring _ 11B in different foraminifera species can reconstruct the upper water column $\mathrm{pH}$ and $\mathrm{pCO} 2$ gradients. I believe this is one of the big strengths of this paper and would like to see an expanded discussion of the depth profiles. One concern is that there may be some circularity here. If the authors have calibrated_11Bforam to modern profiles of

_11Bborate, then by default they would correctly reconstruct the $\mathrm{pH}$ and $\mathrm{pCO} 2$ profile of the water column with the calibration dataset. There are no free parameters.

Response 3: In order to avoid this circularity, we recalculated one calibration for each species excluding the site of interest. Meaning that the recalculated site is not into the calibration. We also use this subset of calibration data to reconstruct vertical profiles in figure 9 which validates the work. When we compare both differences between calibration with or avoiding circularity (figure below or S6), the difference of $\delta 11 \mathrm{~B}$ is on average $(0.8 \%$ on $\delta 11$ Bborate, $0.2 \%$ on $\mathrm{pH}$ and $5 \%$ on $\mathrm{pCO} 2)$. The results are similar to the whole calibration dataset, subset of the data for calibration purposes which validates this work. Maximum divergence can be observed for $\mathrm{G}$. ruber and T. sacculifer when utilizing the calibration [2] and [4]. We also have added a statement to Line 618-620 stating "Reconstruction of seawater $\mathrm{pH}$ and carbonate system parameters is achievable using foraminiferal $\delta 11 \mathrm{~B}$ but additional coretop and down-core studies reconstructing depth profiles will be needed in order to further verify those calibrations."

\section{Detailed review}

RC1: L148-153. The terminology for isotopic fractionation factors and fractionations is incorrect. For Klochko et al. (2006), the fractionation factor, _B, is 1.0272, and the fractionation, "B, is the per mil value of $27.2 \_0.6 \%$ §ËŹ ee, e.g., Table 1 in Farmer et al. (2019) GCA. Please change to correct terminology throughout this paragraph.

Response: Corrected Line 146 "given by the fraction factor $(\alpha)$. The fractionation $(\varepsilon)$ between $\mathrm{B}(\mathrm{OH}) 3$ and $\mathrm{B}(\mathrm{OH}) 4-$ of $27.2 \pm 0.6 \%$ has been empirically determined by Klochko et al., (2006) in seawater."

Printer-friendly version

Discussion paper 
RC1: L166-167. Benthic foraminifera _ 11B are only tangentially relevant to the results of this manuscript, so I recommend deleting this clause and associated references. Unless the benthic _ 11B results directly shed light on your interpretation (as is the case for Amphestegina, below).

Response: I removed the benthic species. Line 160-163: "At present, culture and coretop calibrations have been published for several planktonic species including Trilobatus sacculifer, Globigerinoides ruber, Globigerina bulloides, Neogloboquadrina pachyderma, Orbulina universa (Foster et al., 2008; Henehan et al., 2013; Henehan et al., 2015; Sanyal et al., 1996; Sanyal et al., 2001)."

RC1: L212-224. It may be worth noting here (or perhaps earlier) that this manuscript largely focuses on tropical/subtropical foraminifera.

Response: We have added this information in the abstract (line 50).

RC1: L255. Were the samples dissolved in $1 \mathrm{~N} \mathrm{HCl}$ or $\mathrm{HNO} 3$ ? And why the two different acid matrices? $\mathrm{HCl}$ causes interference issues with ICP-MS measurements. Maybe this does not matter with the microdistillation step, but l'd like to see some explanation (see L260).

Response: The samples were dissolved in $1 \mathrm{~N} \mathrm{HCl}$, results from the microdistillation in order to get the entire dissolution of the sample, including Fe-Mn oxide and hydroxides (reductive acid). Different dissolution after microdistillation resulting in mix $\mathrm{HCl} / \mathrm{HNO} 3$ matrices were tested resulting in no significant $\delta 11 \mathrm{~B}$ differences on standards, however, working with really low concentration samples resulting in running samples in $\mathrm{HCl}$ matrix might be an issue at some point.

RC1: L283-286. Recommend splitting this into two sentences, one on the procedural blanks and one on the acid backgrounds/memory effect.

Response: Lines 279-286: "The sample size for boron isotope analyses typically ranged from $10 \mathrm{ppb} B(\sim 5 \mathrm{ng} \mathrm{B})$ to $20 \mathrm{ppb} B$ samples ( 10 ng $B$ ). Instrumental sen-

Printer-friendly version

Discussion paper 
sitivity for $11 \mathrm{~B}$ was $17 \mathrm{mV} / \mathrm{ppb} \mathrm{B}$ (eg. $170 \mathrm{mV}$ for $10 \mathrm{ppb}$ B) in wet plasma at $50 \mu \mathrm{l} / \mathrm{min}$ sample aspiration rate. Intensity of $11 \mathrm{~B}$ for a sample at $10 \mathrm{ppb}$ B was typically $165 \mathrm{mV}$ $\pm 5 \mathrm{mV}$ closely matched the $170 \mathrm{mV} \pm 5 \mathrm{mV}$ of the standard. Due to the low boron content of the samples extreme care was taken to avoid boron contamination during sample preparation and reduce memory effect during analysis. Procedural boron blanks ranged from $15 \mathrm{pg} B$ to $65 \mathrm{pg} \mathrm{B}$ (contributed to less than $<1 \%$ of the sample signal). The acid blank during analyses was measured at $\leq 1 \mathrm{mV}$ on the $11 \mathrm{~B}$, meaning a contribution $<1 \%$ of the sample intensity, no memory effect was observed within and across sessions."

RC1: L288. As a field, we need to stop considering NEP as a "standard". It is not sufficiently homogenous to be useful for_11B analyses in foraminifera, where precisions of Ân $1 \%$ are absolutely necessary for the vast majority of paleoceanographic purposes.

Response: We agree but due to the specifics of these analytical runs, unfortunately the NEP was the only "relevant" carbonate standard we had. We also ran seawaters as well along with carbonate standards though they did not have the same matrix.

RC1: L349-350 and Figure 4. Please plot the 11Bborate uncertainties on Figure 4. If they are too small to be observed, please note that in the figure caption.

Response: We recalculated the uncertainties based on the errors of Alkalinity and DIC from the Glodap database. Temperature, salinity and pressure were taking into account for all calculations.

RC1: L308-310. I'm surprised that the HF matrix prevents a B memory effect on the Neptune+, but not on the Element XR. To my knowledge, both instruments have effectively the same frontend plasma setup. Can you comment more on this? Does this high $B$ background result from other measurements on the XR, e.g., rock digestions with really high $B$ content? Did you swap out cones, etc? (This comment does not necessarily need to be addressed within the manuscript, I'm just curious).

Printer-friendly version

Discussion paper 
Response: HF matrix does prevent memory effects, but with the Element $\mathrm{XR}$ we are measuring $\mathrm{X} / \mathrm{Ca}$ ratios, so working only in $\mathrm{HF}$ matrix will directly precipitate $\mathrm{CaF} 2$, a mix of $\mathrm{HF}$ and $\mathrm{HNO} 3$ prevents precipitation. The boron background comes from other measurements. The shared instrument is used to run a lot of seawater samples, rocks etc... However, the cones were properly cleaned. We suspect the extraction lengt was the issue. You can still try to decontaminate with HF solution before conditioning the cones but results are not sufficient; One way to decrease the boron background is to condition the cones with the [Ca] standards (HNO3 and HF) for few hours, and switching the boron concentration of the standards.

RC1: L380-382. Except your G. ruber results are not consistent at the low 11Bborate end; they are $1 \%$ o lighter than Henehan et al. (2013) found in sediment traps. It is very important that you state this observation because this is the principal reason for your elevated 11Bforam to 11Bborate slope relative to Henehan and Sanyal.

\section{Response:}

Lines 381-391 "Our results for G. ruber (Fig. 5) are in good agreement with published data from other core-tops, sediment traps, tows, and culture experiments for $\delta 11$ Bborate $>19 \%$ (Foster et al., 2008, Henehan et al., 2013, Raitzsch et al., 2018). However, for $\delta 11$ Bborate $<19 \%$ our results show lighter $\delta 11$ Bcarbonate compared to published values. Whilst this species has been widely studied previously, the sites selected in this study allow us to extend the calibration. The positive offset from the 1:1 curve has been explained by the high photosynthetic activity (Hönisch et al., 2003; Zeebe et al., 2003). Two calibrations have been derived. Utilizing only our data, the sensitivity of $\delta 11 \mathrm{Bcarbonate}$ to $\delta 11 \mathrm{Bborate}$ of our linear regression is not statistically different from 1 and do not follow the low sensitivity trend of the culture experiments from Sanyal et al., (2001) or Henehan et al., (2013), $(p<0.05)$. The uncertainty on this regression is important due to our small dataset and not inconsistent with the second calibration made compiling all data from literature. The sensitivity of this regression is similar (e.g. $0.46( \pm 0.34)$ to the one recently published by Raitzsch et al., (2018) (e.g.

Printer-friendly version

Discussion paper 
It was also highlighted by Michael Henehan (SC1), he was especially asking about the size fraction of our samples, I replied: "Most of the samples have been picked in the 250-300 size fraction (average weight/ shell of $11 \pm 4 \mu \mathrm{g}(\mathrm{n}=4$, SD) only when measurements were realized), we chose a restrained size fraction to avoid this size-related variability (at least in our calibration), we also chose this lower size fraction because some of the sites did not present shells in the higher size fractions and we wanted to stay consistent. Now, when compiling all the data this variability is not constrained. From your paper, our weight/ shell variability could lead to an offset up to $\sim 1 \%$ that we acknowledged can explain most of the variability for our G. ruber data. I have added line 582 "Henehan et al., (2013) reported a lighter $\delta 11 \mathrm{~B}$ with smaller test size, our sample add a weight/shell of $11 \pm 4 \mu \mathrm{g}(\mathrm{n}=4, \mathrm{SD})$ which could also explain this variability."

RC1: L427-439. It could be worth making an updated version of Henehan et al. EPSL 2016's Figure 7 and including this in the main text.

We updated made this figure (below) and added it to the main text.

RC1: L438-439. One option- you could test the influence of sediment core water depth and foraminifer water depth with a multiple linear regression and see if either influence dominates $\mathrm{B} / \mathrm{Ca}$.

Response: At the end, linear regression was only significant when plotting $\mathrm{B} / \mathrm{Ca}$ and the calcification depth not significant with water depth. From those results, the calcification depth is the parameter influencing $\mathrm{B} / \mathrm{Ca}$.

RC1: L457-459 and again L462-463. This is just another way of saying that the depth of the thermocline differs at each location, correct? If so, really what we need are proxies for thermocline depth.

Response: Please see our response to Comment 1.

Printer-friendly version

Discussion paper

RC1: L474-475. Yes, but this might not matter as much for foraminifera_11B. At higher 
latitudes, the seasonality of primary production ( $\&$ hence foraminifera growing seasons) will be more tightly onstrained due to the seasonal progression of winter light limitation and intense vertical mixing and summer nutrient limitation.

Response: Yes, I agree, I changed for:

Line 478-481: "As Raitzsch et al, (2018) highlight, seasonality might be more important at high latitude sites where seasonality is more marked, however, the seasonality of primary production will also be more tightly constrained due to the seasonal progression of winter light limitation and intense vertical mixing and summer nutrient limitation."

RC1:1) You have not yet discussed symbionts in these different foraminifera species until near the end of the section (L509-512). Be explicit about what is known about symbionts in all studies species with an introductory paragraph at the beginning of this section. How biologically similar are the symbiont assemblages in different foraminifera species? Is their concentration, photosynthetic activity, etc. similar? Do they show the same dependence on light intensity to maintain photosynthetic activity? 2) Following on the above, it is not clear to the reader whether "weaker photosynthetic activity" (L487) corresponds to an absence of symbionts, less active symbionts, lower symbiont density, lower light levels, etc. Please clarify. 3) L490. Is symbiont photosynthetic activity a function of light level alone? What about symbiont composition? This comes across as highly speculative without references; please either include references or phrase as a speculation. (In general, it is fine to speculate a little, as long as the reader is aware that you are speculating).

Response: We have expanded the discussion around the types of symbionts/photosynthetic activity/symbiont density. Although there is not a lot of data in the literature, there is a recent study from Takagi et al., (2019) - published this September - that was helpful in strengthening the discussion.

Note it seems that the nutrient concentrations were not impacting the photosynthesis activity for T. sacculifer, and that is why we did not mention it.

Printer-friendly version

Discussion paper 
The following text was added,

Lines 489-501: "In planktonic foraminifera, algal symbiosis is the more common symbiotic relationship. For most of planktonic foraminifera, the host presents only one species of symbionts (Gast and Caron, 2001). The family Globigerinidae, including G. ruber, T. sacculifer and O. universa, commonly have dinoflagellates or chrysophyte algal symbionts (Anderson and Be, 1976; Spero, 1987). The families Pulleniatinidae, Globorotaliidae, including N. dutertrei, P. obliquiloculata, G. menardii and G. tumida, have chrysophyte algal symbionts (Gastrich, 1988). The relationship between the symbionts and the host is complex by nature. Nevertheless, this symbiotic relationship provides energy (Hallock, 1981b) and promotes calcification of the foraminifera (Duguay, 1983; Erez et al., 1983) by providing the inorganic carbon to the host (Jorgensen et al., 1985). Also, for T. sacculifer and O. universa photosynthesis increases with higher insolation (Jorgensen et al., 1985; Rink et al., 1998). Dinoflagellate-bearing foraminifera (G. ruber, T. sacculifer and O. universa) tend to have a higher symbiont density and photosynthesis activity while P. obliquiloculata, G. menardii and N. dutertrei have lowered symbiont density and P. obliquiloculata, N. dutertrei lower photosynthetic activity (Takagi et al., 2019). P. obliquiloculata showed the minimum symbiont density and photosynthetic activity (Takagi et al., 2019)."

Lines 526-530: "Also, T. sacculifer has the potential to support more photosynthesis due to its higher symbiont density. Higher photosynthetic activity is observed compared to other species potentially supporting higher symbiont/host interactions. Those results could be in line with a greater sensitivity of $\mathrm{T}$. sacculifer photosynthetic activity with changes in insolation/water depth. It can also be noted that this species presents the most important variations in symbiont density versus its test size. Microenvironment $\mathrm{pH}$ results for $\mathrm{N}$. dutertrei, $\mathrm{G}$. menardii, $\mathrm{G}$. tumida, are similar to O. universa and suggest a threshold for respiration driven $\delta 11 \mathrm{~B}$ signature. This threshold can be driven by a change of photosynthetic activity due to lower light intensity at deeper depth and/or a change in the symbiont assemblage with non-dinoflagellate symbionts

Printer-friendly version

Discussion paper
Interactive comment 
at deeper depth. We can explain this threshold because deep dweller species do not experience important changes of insolation at those depths so their microenvironments should be respiration driven and relatively stable. We can also note that P. obliquiloculata which has the lowest symbiont density and photosynthetic activity has the lowered microenvironment $\mathrm{pH}$ compared to other deeper dweller species supporting this respiration driven microenvironment."

$\mathrm{RC1}$ : 4) Light intensity in the ocean is not a function of water depth alone; turbidity matters quite a lot. Is it reasonable to compare the light intensity in different oceanographic regions as a function of water depth alone?

Response: This is true, but we think it is still reasonable for the calculations because we used a light attenuation coefficient from Wang et al., (2008) which should take most of the parameters into account even if no variability is constrained.

RC1: 4) L492. The negative relationship between_11B and water depth in Figure S2 is driven only by the low_11B measured in western equatorial Pacific T. sacculifer. Have you propagated your uncertainty in depth habitat to _11Bborate in your calculation of 11B as plotted in Figure S2? Looking at the western equatorial Pacific (WP7-01), the 80_20m depth range from CD2 corresponds to a _1\%。_11Bborate range (Fig. 4). (If you include the CD3 estimate of 125_15 m, the total possible _11Bborate increases to $1.5 \%$ Add on the _ 11 Bforam measurement uncertainty of $\_.22 \%$ and I cannot see how you get a total_11B uncertainty of $<1 \%$ 。

Response: The uncertainties on the $\delta 11$ Bborate are calculated using Michael Henehan's code which takes into account the uncertainties on $\mathrm{pH}$, temperature, salinity and $\delta 11$ Bseawater. Uncertainties of $\mathrm{pH}$, temperature and salinity were calculated integrating the parameters between uncertainties of the calcification depth. This is the same approach that was used in Raitzsch et al. (2018). Then the uncertainty on $\Delta 11 \mathrm{~B}$ was measured by the square root of the sum of the square uncertainties on $\delta 11$ Bborate and $\delta 11$ Bcarbonate.

Printer-friendly version

Discussion paper 
$\mathrm{RC1:} 5)$ I do not think linear regression is the appropriate test statistical test for the significance of the _11B-water depth relationship, because depending on how you performed the regression, it may not account for the uncertainty on each datapoint. $A$ t-test for mean difference between Arabian Sea, Indian Ocean, and western equatorial Pacific T. sacculifer_11B would be more robust. I would bet that such a test will indicate no significant _11 11 difference between the different regions given the small sample sizes. If true, this detailed line of discussion is unnecessary; instead, you can present this as an observation that requires future study to confirm or deny.

Response: We have added two data points from sites $806 \mathrm{~A}$ and $807 \mathrm{~A}$ which makes the t-test more robust.

For $\delta 11 \mathrm{~B}$. Unpaired t-test between the WEP and the rest of the samples for T. sacculifer (w/o sacc) is significantly different $(p=0.01)$. For $\Delta 11 \mathrm{~B}$. Unpaired t-test between the WEP and the rest of the samples for T. sacculifer (w/o sacc) is not significantly different $(p=0.067)$. If now we look at both $G$. ruber and T. sacculifer (w/o sacc) which are presented in the plot, difference between Arabian Sea+Indian Ocean and WEP is significantly different $(p=0.0075)$. If we also look at $T$. sacculifer (w/o sacc and sacc) + G.ruber between Arabian Sea+Indian Ocean and WEP the difference is significantly different $(p=0.0427)$.

Lines 520-522: "Especially, we observe an important decrease of $\delta 11 \mathrm{~B}$ in the WEP for T. sacculifer (w/o sacc), significantly different from the other sites $(p<0.05)$ and a calculated $\triangle 11 \mathrm{~B}$ of $\mathrm{G}$. ruber, T. sacculifer (w/o sacc and sacc) significantly lower in the WEP compared to the other sites $(p<0.05)$."

$\mathrm{RC1}$ : Any precipitation rate implications need to be very carefully phrased. The higher "values" for G. ruber and T. sacculifer have large uncertainties, so they are probably not robustly higher than other species (unless a statistical test confirms this to be true). Moreover, it is unclear the extent to which the growth rates of different foraminifera species differ from one another. Nevertheless, your point on higher B/Ca sensitivity to

Printer-friendly version

Discussion paper 
borate/bicarbonate in the shallower species is very interesting.

Response: We mitigated this paragraph.

Lines 556-567: "When combining all literature data, T. sacculifer and G. ruber have sensitivities of $\delta 11$ Bcarbonate to $\delta 11$ Bborate of $0.83 \pm 0.48$ and $0.46 \pm 0.34$ respectively in line with previous literature and paleo-CO2 reconstructions. Also, if we only take into account our data, the observation that the sensitivity of $\delta 11$ Bcarbonate to $\delta 11$ Bborate are not statistically different from unity for most of the species investigated we can speculate that for these taxa, changes in precipitation rate and contributions of boric acid are not likely to be important. If considering only the data from this study, G. ruber (1.12 \pm 1.67$)$ and T. sacculifer $(1.38 \pm 1.35)$ present higher sensitivities of $\delta 11$ Bcarbonate to $\delta 11$ Bborate. We can then again speculate that the observed high values for $\delta 11$ Bcarbonate at high seawater $\mathrm{pH}$ can be due to higher precipitation rates. We note this could also be consistent with the higher sensitivity of $\mathrm{B} / \mathrm{Ca}$ signatures in these two surface dwelling species to ambient $[\mathrm{B}(\mathrm{OH}) 4-] /[\mathrm{HCO} 3-]$ relative to deeper dwelling species. As indicated by Farmer et al., (2019), studies of calcite precipitation rates in foraminifera could help to test this hypothesis and improve our understanding of the fundamental basis of boron-based proxies."

RC1: L525-527. What do you mean by "higher values "here? Slopes of $\delta 11$ Bforam to $\delta 11$ Bborate regressions, or $\Delta 11 \mathrm{~B}$, or something else?

Response: Lines 561-562:" G. ruber (1.12 \pm 1.67$)$ and T. sacculifer (1.38 \pm 1.35$)$ present higher sensitivities of $\delta 11$ Bcarbonate to $\delta 11$ Bborate."

RC1: L541-542. Given the speculative nature of the depth/light effect on symbiont photosynthesis, foraminifer microenvironment $\mathrm{pH}$ and thus foraminifera _11B, change to "which may be explained by the deeper depth habitat for these taxa in the WEP, where lower light levels might reduce symbiont photosynthetic activity". Or remove.

Response: Changed.

Printer-friendly version

Discussion paper 
RC1: Fig.2/Fig.4

Response: Front made bigger

$\mathrm{RC1}$ : Figure 7 (or below). I really cannot follow this figure at all. Should there be a $\mathrm{pH}$ axis?

Response: For more clarity, we combined in Figure 8 (or below) the $\Delta$ microenvironment $\mathrm{pH}$ and this depth profile. This depth profile only aims to explain the low $\delta 11 \mathrm{~B}$ in the WEP. This is based on the calculation of insolation needed to explain the decrease in $\Delta \mathrm{pH} 0, \Delta \mathrm{pH} 1$ and $\Delta \mathrm{pH} 2$ observed from the $\delta 11 \mathrm{~B}$. This is an independent way to calculate the depth habitat explaining the $\delta 11 \mathrm{~B}$. The insolation is calculated based on Jorgensen et al., (1985). I found the insolation needed to decrease of $\Delta \mathrm{pH}, \Delta \mathrm{pH} 1$ and $\Delta \mathrm{pH} 2$ and converted in \% insolation in the water column. What is interesting is that the decrease of insolation around $125 \mathrm{~m}$ can explain the low $\delta 11 \mathrm{~B}$ values.

Please also note the supplement to this comment: https://www.biogeosciences-discuss.net/bg-2019-266/bg-2019-266-AC1supplement.pdf

Interactive comment on Biogeosciences Discuss., https://doi.org/10.5194/bg-2019-266, 2019. 


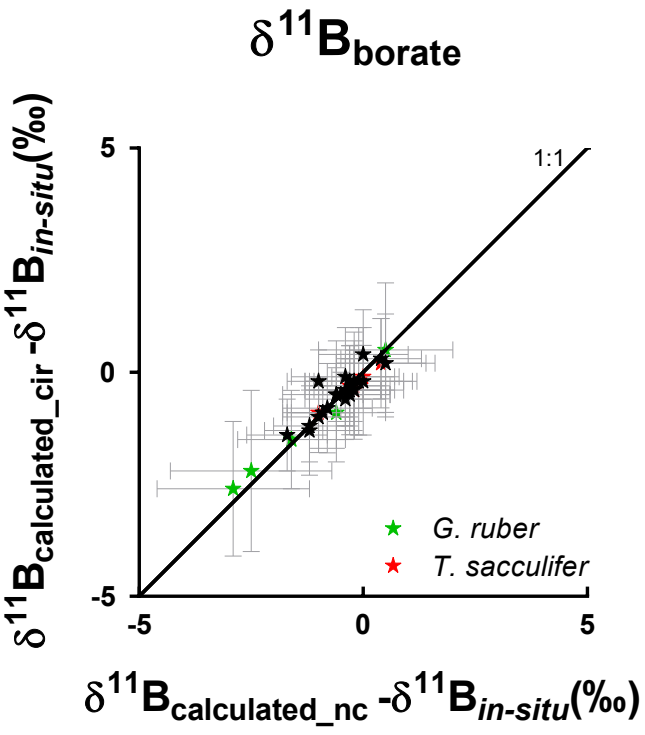

\section{pH}

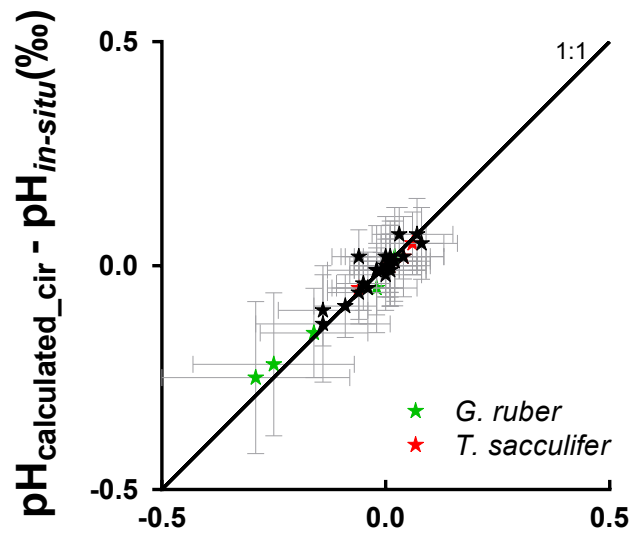

$\mathrm{pH}_{\text {calculated_nc }}-\mathrm{pH}_{\text {in-situ }}(\% \mathrm{o})$

Fig. 1. 


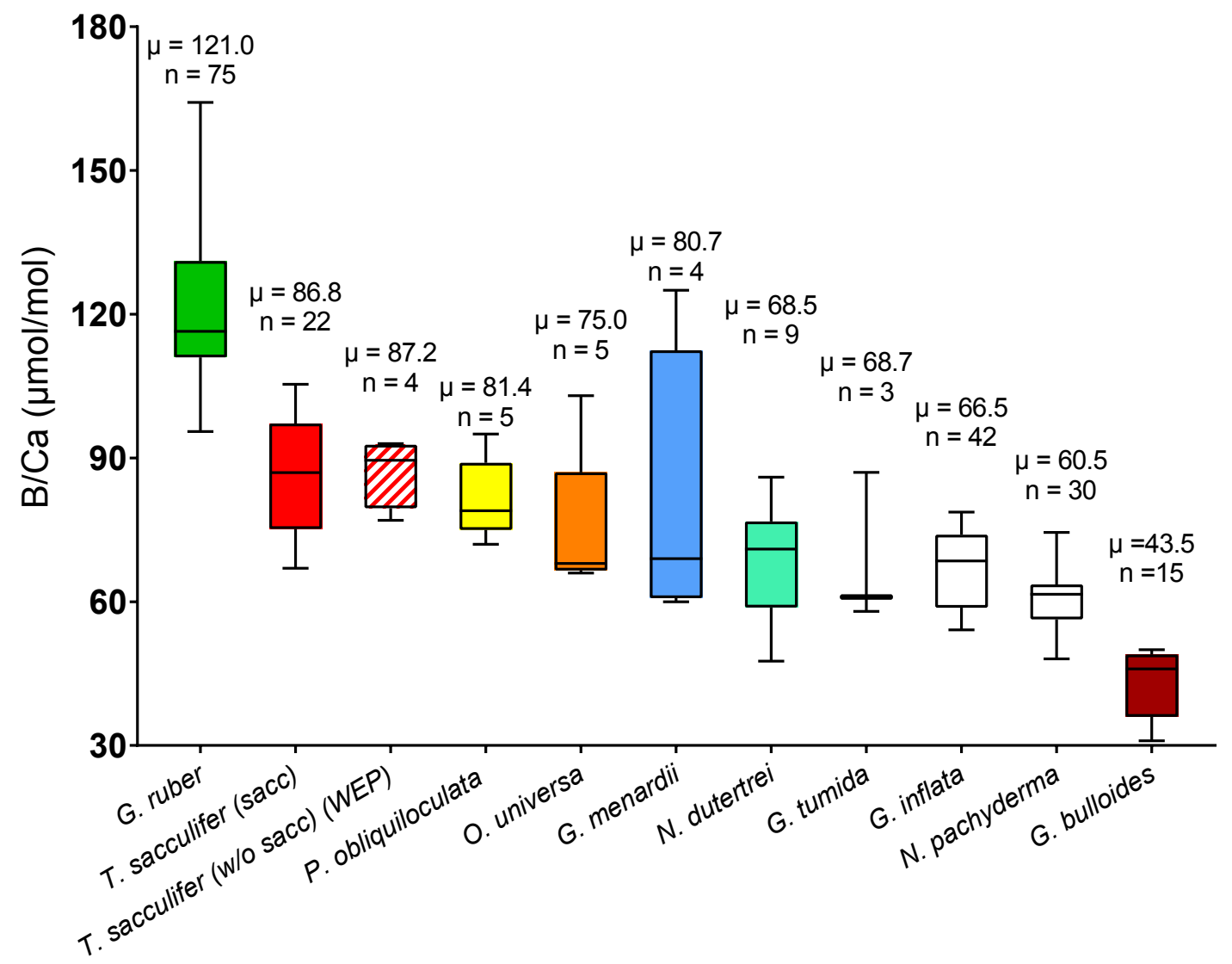

Interactive

comment

Fig. 2. 
Interactive

comment

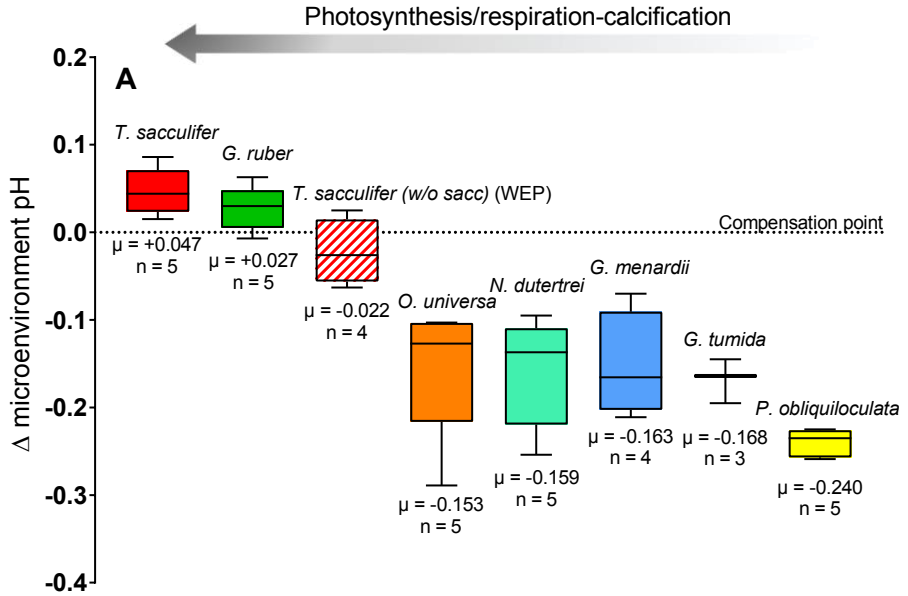

B

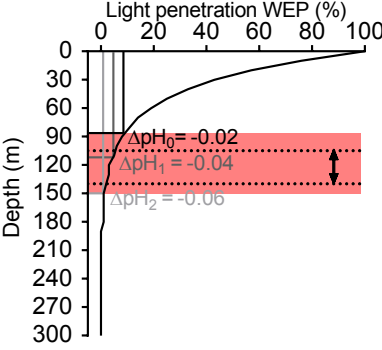

Fig. 3.

Printer-friendly version

Discussion paper 